\title{
A model for rockfall protection structures based on a multi-scale approach
}

\author{
F. Bourrier ${ }^{1}$, P. Gotteland ${ }^{1}$, F. Nicot ${ }^{2}$, and S. Lambert ${ }^{2}$ \\ ${ }^{1}$ L3S-R ; UJF, INPG, CNRS ; Grenoble Universités - BP 53 - 38041 Grenoble \\ Cedex 9 - France ; email : philippe.gotteland@ujf-grenoble.fr ; \\ franck.bourrier@hmg.inpg.fr \\ ${ }^{2}$ Cemagref, UR ETGR, F-38402 St-Martin d'Hères, France; email : \\ francois.nicot@cemagref.fr ; stephane.lambert@cemagref.fr
}

\begin{abstract}
Cellular sandwich structures constitute efficient technological solutions to protect elements at risk from rockfall hazard. The design of these structures is for the most part empirically based which does not allow optimization. The mechanical behaviour of a cellular structure is investigated using a multi-scale approach: from the individual layers of the structure made up of rocky particles contained in wire netting cages, fine granular material and reinforced embankment, to the entire structure which is an assembly of these different layers. A computational tool based on discrete modelling of the structure is developed for design purposes. Information is gathered from experiments at the layer scale to calibrate the parameters of the structure model. These experimental results are also used to evaluate the capability of the model to provide relevant results for the impact of falling rocks on the structure.
\end{abstract}

\section{INTRODUCTION}

Reinforced soil structures can be used to protect infrastructures against rockfall. The technologies used to build such structures lead to massive structures, i.e. embankments, built from geomaterials, and located upstream in the area potentially affected by the falling rocks (Labiouse et al., 1994; Hearn et al., 1995; RiskYdrogeo, 2006; Peila et al., 2007; Aminata et al., 2008). To reduce the overall size of such protection structures and improve their efficiency, innovative technologies using cellular sandwich structures are developed (Figure 1). These structures are composed with several layers: a front layer impacted by the falling boulder coupled to a kernel layer aiming at dissipating energy and a back part that maintains structure stability and increases structure rigidity. The front layer is composed of coarse, gravel-like materials contained in wire netting cages, or geocells. The kernel layer is composed of fine granular materials that can either be sand or sand mixed with scrapped tyres (Gotteland et al., 2007). Finally, the back part can either be a rigid concrete wall or a reinforced soil embankment.

The aim of this study is to develop a practical design tool for rockfall protection sandwich structures using a multi-scale approach (Bertrand et al., 2006; Nicot et al., 2007). Multi-scale approaches consist in collecting information at the elementary scale, called microscopic scale, and at an intermediary scale, called mesoscopic scale, to define a model usable at the macroscopic scale. Multi-scale approaches therefore make it possible to investigate the response of a structure on a macroscopic scale by accounting for the determinant properties of constitutive 
materials on both microscopic and mesoscopic scales. In this study, the microscopic scale corresponds to the size of the particles of the materials of the structure, the mesoscopic scale is associated with each layer of the structure and the macroscopic scale is the structure scale. The approach presented consists in defining constitutive models for each layer type from experiments at the mesoscopic scale in order to couple these models and define a macroscopic structure model. The principle of the macroscopic numerical model of the structure is first presented. The calibration of the constitutive models for each layer type is then exposed. Finally, comparisons between impact simulations and experimental results are performed for impacts on individual cells and on layers assembly.

\section{MULTI-SCALE MODELLING OF A SANDWICH STRUCTURE}

\section{Principle of the structure model}

The type of structure considered is presented in Figure 1.The size of the geocells of the front layer is $50 \times 50 \times 50 \mathrm{~cm}$.

This structure is modelled as an assembly of cells. The geometries of the front and kernel layers are explicitly modelled whereas the back part embankment is modelled as an elastic boundary condition (Figure 1). The kernel layer is divided into cells of same size as the front layer geocells. The cells are modelled by spherical particles located at their centre. In a first approximation, it is assumed that each cell can only move along the normal direction to the front face of the structure ( $x$ direction). Indeed, due to the confinement of geocells within the structure, it seems reasonable to consider the displacements of the gravity centre of the cells along both $y$ - and $z$-directions negligible with respect to the displacements along $x$-direction.
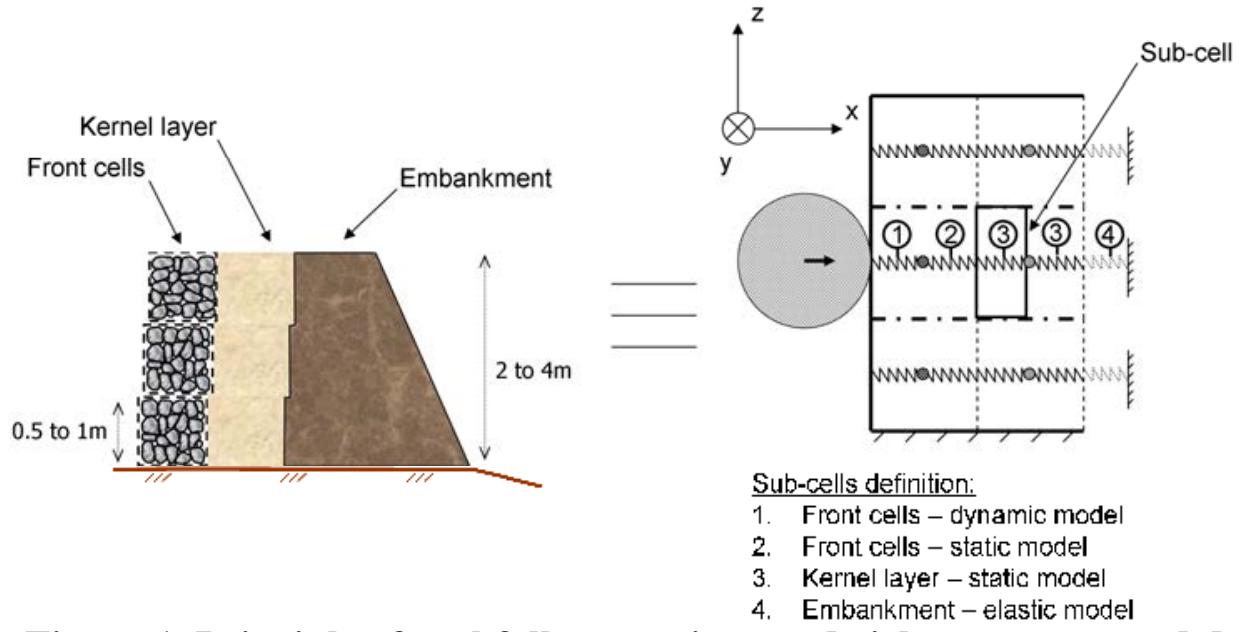

Figure 1. Principle of rockfall protection sandwich structures and definition of the structure model.

The different cells of the structure interact at their interfaces by means of forces applied at the cells gravity centre. For the calculation of the interaction forces, each cell is divided into two sub-cells and a constitutive model is associated with each of these sub-cells (Figure 1). Depending on the sub-cells position, the 
constitutive models are developed from dynamic or static experimental investigations. Front sub-cells that are directly impacted by the boulders require defining constitutive models from experiments in which sub-cells are subjected to dynamic loadings (sub-cell 1 on Fig. 1). On the contrary, inner sub-cells inside the structure are considered to be only subjected to static loadings.

\section{Front sub-cells: dynamic constitutive models}

As the impacting boulder stiffness is larger than the front sub-cell stiffness the interaction force between them is only depending on the sub-cell strain. In the $x$ direction, the increment $d F_{i}$ of the interaction force $F_{i}$ between the boulder and the cell $i$ of the front layer is given by (Figure 2):

$d F_{i}=k_{i}^{g} d u_{i}$

where $d u_{i}$ is the increment of the penetration of the boulder inside the cell $i$ :

$d u_{i}=d x_{r}-d x_{i}$

where $k_{i}^{g}$ is the stiffness of the front sub-cell of the cell $i$. The stiffness $k_{i}^{g}$ is defined from a constitutive model based on the function $f^{d}{ }_{i}$ which relates the force $F_{i}$ on the projectile and the projectile penetration $u_{i}$ :

$k_{i}^{g}=\frac{\partial f_{i}^{d}}{\partial u_{i}}$

As the impact direction of the velocity of the boulder is not normal to the front face of the structure, a tangential force at the contact point also exists. This force is depending on the normal force $F_{i}$ following a Coulomb's friction model. Modelling this tangential interaction allows accounting for the changes in the boulder tangential and rotational velocities during the impact.

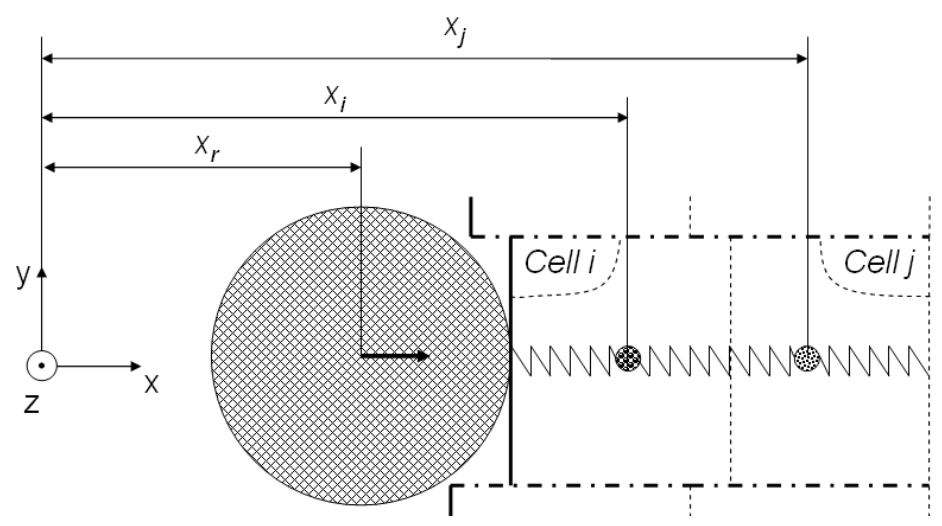

Figure 2. Cell strains definition. 
The experiments (Lambert et al., 2009) used to characterize the constitutive models consisted in the impact of cells located above a concrete support by a spherical boulder. The lateral boundary conditions of the impacted cells (Figure 4) were either free-to-deform (FD), confined with the same material as the fill material of the cell (MC) or rigidly confined (RC).

In accordance with the experimental results (Figure 5a) from Lambert et al. (2009), different patterns of the constitutive models of the front sub-cells are defined depending on the lateral boundary conditions (Figure 5b). For any boundary conditions, the same linear relation is modelled for penetrations smaller than $u^{\text {lim }}$ : $F_{i}=k^{l} u_{i}$. For penetrations larger than $\mathrm{u}^{\lim }$, the same linear relation is used for RC conditions. On the contrary, the impact force remains constant for both FD and MC conditions. However, the value of the force $F_{i}$ is lower for FD conditions $\left(F^{p l}\right)$ than for MC conditions $\left(F^{l i m}\right)$. Finally, for any confining conditions, the unloading phase is characterized by a strong linear decrease of the force $F_{i}$ for decreasing penetrations:

$F_{i}=k^{u l} u_{i}$

The calibration of all parameters will be exposed in details in the section dedicated to the simulation results.

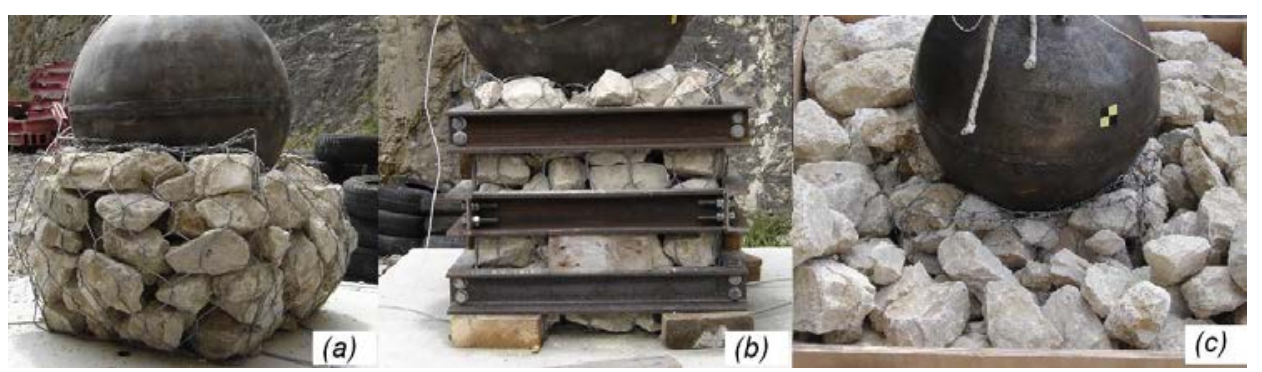

Figure 4. Lateral boundary conditions used in the experiments: a) free-todeform (FD) ; b) rigid confinement (RC) ; c) material confined (MC) (after Lambert et al., 2009).

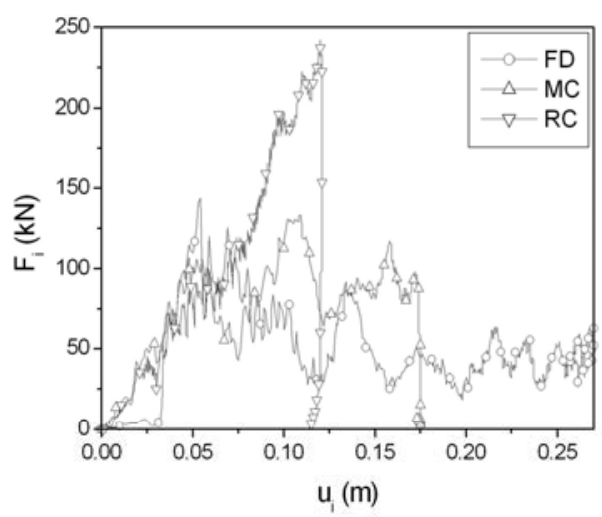

a)

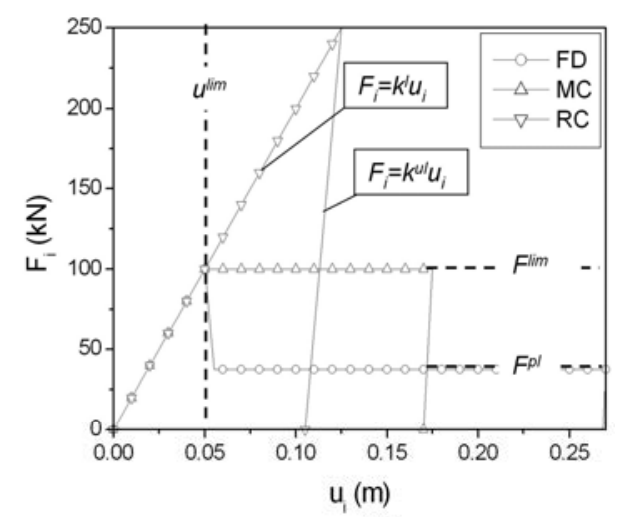

b)

Figure 5. Experimental results of impacts on front- sub-cells (a) and corresponding constitutive models (b). 


\section{Inner sub-cells: static constitutive models}

The interaction force between inner sub-cells depends on the strains of both sub-cells in contact along the $x$-axis. The equilibrium condition between the two adjoining sub-cells $i$ and $j$ yields a relation between the increment $d F_{i j}$ of the interaction force $F_{i / j}$ and the relative displacement $d u_{i j}$ between the two cells along the x-direction, (Figure 2):

$d F_{i / j}=\frac{S_{c} E_{i j}}{l_{i}^{d 0}+l_{j}^{g 0}} d u_{i j}$

with

$d u_{i j}=d x_{j}-d x_{i}$

where $l^{d 0}{ }_{i}, l^{g 0}{ }_{j}$ are the lengths of the sub-cells associated with the cell $i$ and cell $j$ at the first time step ; and $S_{c}$ is the area of the interaction surface. $E_{i j}$ is the equivalent modulus accounting for both modulii $E^{d}{ }_{i}$ and $E^{g}{ }_{j}$ of the sub-cells:

$$
E_{i j}=\frac{E_{i}^{d} E_{j}^{g}}{E_{i}^{d}+E_{j}^{g}}
$$

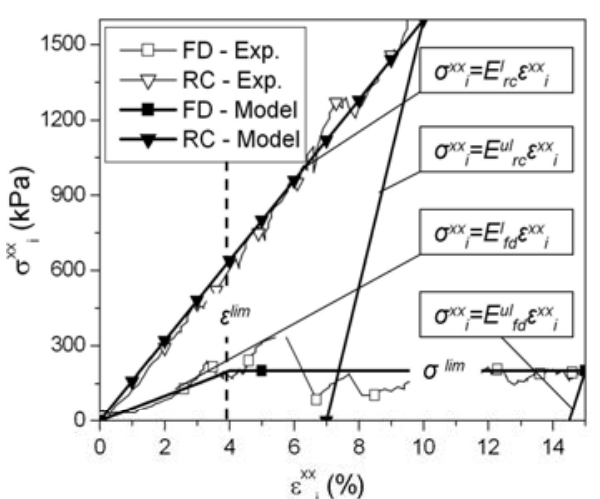

a)

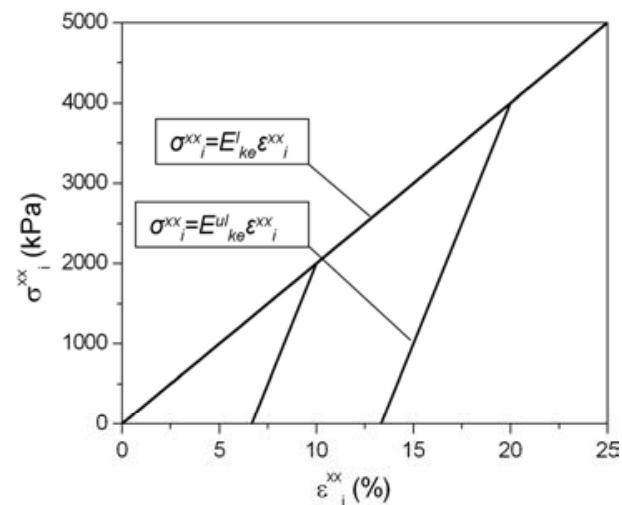

b)

Figure 6. Constitutive models for inner sub-cells associated with front cells (a) and for inner sub-cells associated with kernel cells (b).

Assuming that the interface between adjoining cells exhibits only little changes in size and shape over the loading, it is possible to express the modulii $E^{d}{ }_{i}$ and $E^{g}{ }_{j}$ in terms of stresses and strains. For inner sub-cells associated with the front layer, the constitutive models are characterized from static compression tests (Lambert, 2007) of cells under RC and FD conditions (Figure 6). For FD conditions, the evolution of the axial stress $\sigma^{x x}{ }_{i}$ between sub-cells in the $x$-direction, depending on the sub-cell axial strain $\varepsilon^{x x}{ }_{i}$ in the $x$-direction, is characterized by a linear increase in the stress until the threshold value $\varepsilon^{\lim }$ is reached. For $\varepsilon^{x x}{ }_{i}$ larger than $\varepsilon^{\lim }$, the stress is equal to $\sigma^{l i m}$. On the contrary, no threshold value is observed for RC conditions. 
Interestingly, the coefficient of the linear relation is different depending on the confinement conditions (Figure 6a): $E_{r c}^{l}$ (for RC conditions) and $E_{f d}^{l}$ (for FD conditions). Finally, the unloading phase is also characterized by a linear decrease of $\sigma^{x x}{ }_{i}$ for decreasing $\varepsilon^{x x}{ }_{i}$ using the coefficient $E^{u l}{ }_{r c}$ for RC conditions, and $E^{u l}{ }_{f d}$ for FD conditions (Figure 6a). The constitutive model associated with MC conditions is comprised between these two extreme cases. Values of the parameters obtained are reported in Table 1.

For the kernel layer, it is assumed that the constitutive behaviour of sub-cells can be modelled as a bilinear relation involving a loading modulus $E_{k e}^{l}$ and an unloading modulus $E^{u l}{ }_{k e}$ (Figure 6b).

Finally, the back part of the structure is associated with a linear elastic constitutive model characterized by the elastic modulus $E_{b o}$.

\section{Lateral forces}

The cell strain in the $x$-direction entails lateral cell strains that induce normal forces $F^{l a t}{ }_{i}$ at cell interfaces in the $y$ - and $z$-directions. $\mathrm{F}^{\text {lat }}{ }_{i}$ are calculated with $K_{\delta}$, a constant coefficient, as follows (Nicot et al., 2007):

$$
F_{i}^{l a t}=K_{\delta}\left(l_{i}^{g}+l_{i}^{d}\right)
$$

where $\mathrm{l}_{\mathrm{i}}^{\mathrm{d}}, \mathrm{l}_{\mathrm{j}}^{\mathrm{g}}$ are the lengths of the sub-cells associated with the cell $i$ and cell $\mathrm{j}$ at the current time step.

The $F^{\text {lat }}{ }_{i}$ forces induce tangential forces $F_{i}^{f}$ that counter the cells displacements along the $\mathrm{x}$-direction:

$$
F_{i}^{f}=\mu_{i} F_{i}^{\text {lat }}
$$

where $\mu_{i}$ is the Coulomb's friction coefficient associated with the cell $i$.

The values of $K^{\delta}$ and $\mu_{i}$ were determined from numerical simulations performed at the cell scale (Nicot et al., 2007). One can also note that, when the cell length reaches a limit value $l^{\text {lim }}$ corresponding to the compaction limit of the cell, the cell is considered as a rigid body that stills interacts with its neighbouring cells.

\section{IMPACT SIMULATIONS}

All simulations were done using an explicit solving scheme. At the beginning of the simulation, the velocity of the boulder is set at the desired value. For each time step, the structure model used allows calculating the position, the velocities and the interaction forces applied on the impacting boulder and on all cells.

Simulations of impacts at the cell scale are first held to calibrate the parameters of the constitutive models for the sub-cells associated with the front layer. The model considered is simplified as in this case there is no kernel (Figure 7). The calibration procedure consisted in comparing the experiments (Lambert et al., 2009) with the simulations allowing calibrating the dynamic constitutive model of the front sub-cells for different lateral boundary conditions (Figure 8). The impact force measured for MC conditions is first used to calibrate the parameters $u^{\lim }, k^{l}, k^{u l}$ and 
$F^{l i m}$ (Table 1). Once these parameters are fixed, simulations under FD conditions allow calibrating the parameter $F^{p l}$.
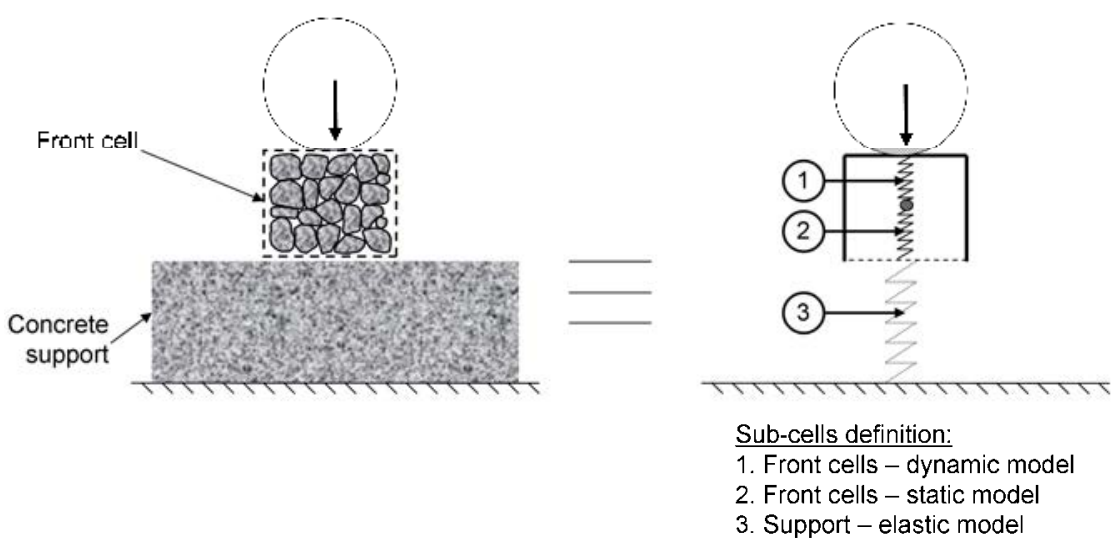

Figure 7. Model used for the simulations of impacts at the cell scale.

Table 1. Values of the parameters of the constitutive models.

\begin{tabular}{l|l|l}
\hline \multirow{2}{*}{$\begin{array}{l}\text { Dynamic constitutive model } \\
\text { Front layer }\end{array}$} & $F D$ & $\begin{array}{l}u^{l i m}=0.05 \mathrm{~m} ; k^{l}=2 \times 10^{6} \mathrm{~N} / \mathrm{m} ; k^{u l}=2 \times 10^{7} \mathrm{~N} / \mathrm{m} ; F^{l i m}=90000 \mathrm{~N} / \mathrm{m} ; \\
F^{p l}=37000 \mathrm{~N}\end{array}$ \\
\cline { 2 - 3 } & $M C$ & $u^{l i m}=0.05 \mathrm{~m} ; k^{l}=2 \times 10^{6} \mathrm{~N} / \mathrm{m} ; k^{u l}=2 \times 10^{7} \mathrm{~N} / \mathrm{m} ; F^{l i m}=90000 \mathrm{~N} / \mathrm{m}$ \\
\hline \multirow{2}{*}{$\begin{array}{l}\text { Static constitutive model } \\
\text { Front layer }\end{array}$} & $F C$ & $k^{l}=2 \times 10^{6} \mathrm{~N} / \mathrm{m} ; k^{u l}=2 \times 10^{7} \mathrm{~N} / \mathrm{m}$ \\
\cline { 2 - 3 } $\begin{array}{l}\text { Static constitutive model } \\
\text { Kernel layer }\end{array}$ & $R C$ & $E^{l i m}=36 \mathrm{MPa} ; E^{u l}{ }_{r c}^{l i m}=360 \mathrm{MPa} ; \sigma^{l i m}=60 \mathrm{kN} / \mathrm{m}^{2} ; E_{f d}^{l}=2 \mathrm{MPa} ; E_{f d}^{u l}=20 \mathrm{MPa}$ \\
\hline $\begin{array}{l}\text { Elastic boundary conditions } \\
\text { Back part }\end{array}$ & $E_{k e}^{l}=200 \mathrm{MPa} ; E^{u l}{ }_{k e}=600 \mathrm{MPa}$ \\
\hline
\end{tabular}

The comparison between the experiments and the simulations for the time evolution of the impact force and of the force transmitted to the concrete support first shows the capability of the model to capture the dynamic effects occurring during the impact (Figure 8). The time delay between the peak of the impact forces and of the transmitted forces is very similar and the curves also exhibit similar global shapes. On the other hand, the model is not able to reproduce the noises observed on the experimental curves resulting from the stones crushing (Lambert et al., 2009). Indeed, the model considers the cell as a unique element whereas it is made of a large number of stones that successively crush. This also explains the poor matching for FD curves. From a quantitative point of view, the peak values reached are also of the same order both in the experiments and in the simulations for any lateral boundary conditions. One could however note a slight underestimation of the peak values of the transmitted forces in the simulations. This quantitative accordance is not surprising for what concerns the impact forces because the parameters of the model were calibrated in this purpose. On the contrary, the experimental results for the transmitted forces were not used in the calibration procedure. The accordance 
between the experiments and the simulations for the time evolution of the transmitted forces therefore constitutes a validation of the model (Figure 8).

a)

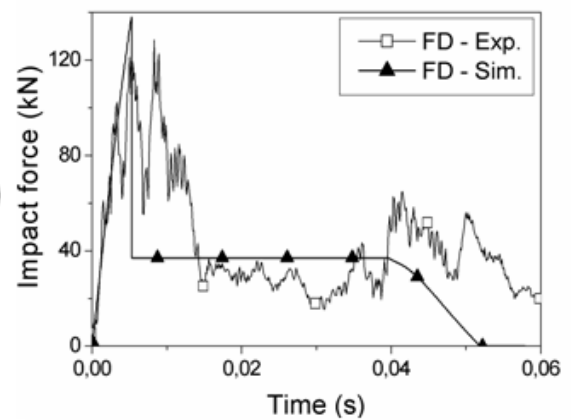

b)
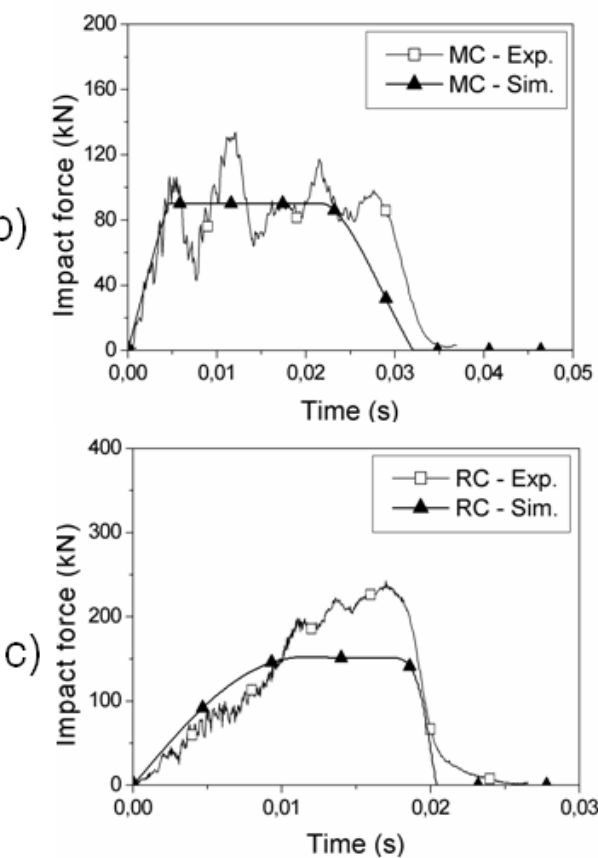
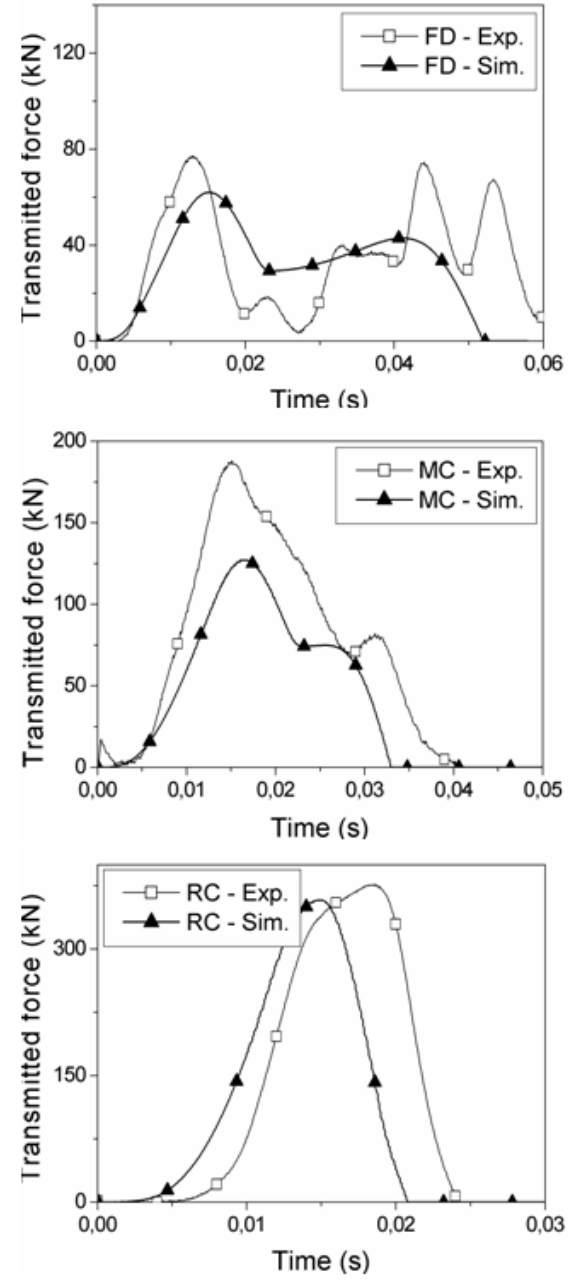

Figure 8. Comparison between experiments and simulations for impacts on front cells under FD (a), MC (b) and RC (c) conditions.

In a second phase, experiments of the impact of a boulder on a RC front cell located above a $50 \mathrm{~cm}$ sand layer were simulated using the structure model (Figure 9). These simulations aimed at calibrating both the loading and the unloading modulus of the constitutive model associated with kernel cells (Table 1).

The results of this calibration procedure show that relevant quantitative values of the maximum impact and transmitted forces can be obtained using the structure model. These results also show that using a simple bilinear constitutive model for the sub-cells associated with the kernel layer does not allow precisely accounting for the time evolution of the transmitted force, in particular (Figure 10). Here again, the experimentally observed noises are not reproduced. Using more complex constitutive models could improve the model on this point. However, the calibration of such constitutive models would require using complementary experimental results that are not yet available. 

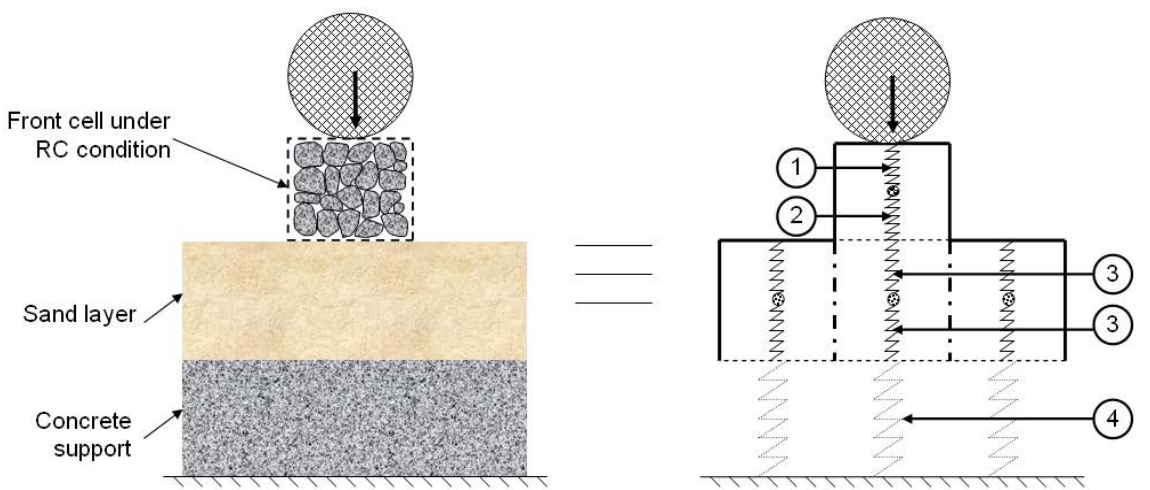

Sub-cells definition:

1. Front cells - dynamic model - RC conditions

2. Front cells - static model - RC conditions

3. Kernel layer - static model

4. Support - elastic model

Figure 9. Model used for the simulation of impacts on a front cell under $\mathrm{RC}$ conditions located above a $50 \mathrm{~cm}$ sand layer.
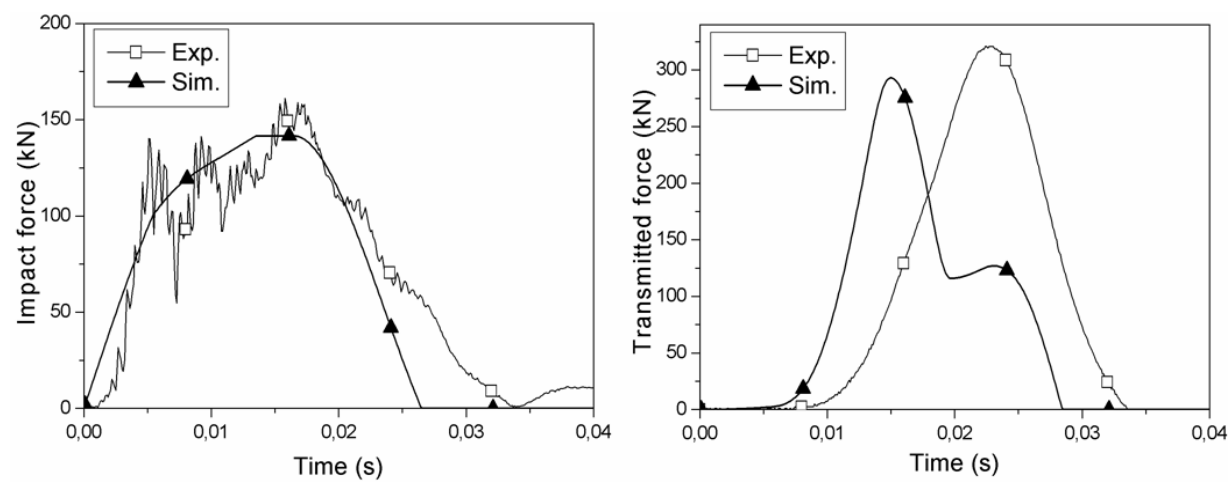

Figure 10. Comparison between experiments and simulations for the impact on a front cell under RC conditions located above a sand layer.

\section{CONCLUSION}

In this paper a model usable for the design of rockfall protection sandwich structures is proposed. This model is based on a multi-scale approach which allows getting information from experiments at a mesoscopic scale, the cell scale, in order to characterize the parameter of a macroscopic model, the structure model.

This paper focuses on describing the structure model and the procedure for the calibration of the parameters of this model. The simulations of impact experiments using a simplified version of the structure model provide encouraging results regarding the capability of the model to capture the mechanisms occurring during the impact. However, the constitutive models associated with the kernel layers should be improved in order to increase the capability of the model to account for dynamic mechanisms occurring during the impact. In addition, in the near future, the capability of the model to be used for the design of real-scale structures will be evaluated using results of impact experiments on real-scale sandwich structures that are currently in progress. 


\section{ACKNOWLEDGEMENTS}

The results presented in this paper were obtained in the framework of the French research development project REMPARe (www.rempare.fr) supported by the French National Research Agency (ANR). All the partners of project REMPARe as well as, for their financial support, the research consortium VOR-RNVO, and the PGRN (Natural Hazard Pole of Grenoble) from the Isère General Council are gratefully acknowledged by the authors.

\section{REFERENCES}

Aminata, D., Yashima, A., Sawada, K., Sung, E., Sugimori, K., and Inoue, S. (2008). "New Protection Wall Against Rockfall Using a Ductile Cast Iron Panel." Journal of Natural Disaster Science, 30 (1): 25-33.

Bertrand, D., Nicot, F., Gotteland, P., and Lambert, S. (2006). "Modelling a geocomposite cell using discrete analysis." Computers and Geotechnics, 32: 564577.

Gotteland, P., Lambert, S., and Salot, C. (2007). "Investigating the Strength characteristics of tyre chips - sand mixtures for geo-cellular structure engineering." Proc. Scrap Tire Derived Geomaterials - Opportunities and Challenges, Yokosuka: 351-361.

Hearn, G., Barrett, R., and Henson, H. (1995). "Development of effective rockfall barriers." Journal of transportation engineering, 121 (6): 507-516.

Labiouse, V., Descoeudres, F., Montani, S., and Schmidhalter, C. (1994). "Experimental study of rock blocks falling down on a reinforced concrete slab covered by absorbing cushions." Revue française de géotechnique, 69: 41-61.

Lambert, S. (2007). Comportement mécanique de géo-cellules - application aux constituants de merlons pare-blocs cellulaires. PhD thesis, Université Joseph Fourier, Grenoble, France, 237 p (in french).

Lambert, S., Gotteland, P., and Nicot, F. (2009). "Experimental study of the impact response of geocells as components of rockfall protection embankments." Natural Hazards and Earth Systems Sciences, 9: 459-467.

Nicot, F., Gotteland, P., Bertrand, D., and Lambert, S. (2007). "Multi-scale approach to geo-composite cellular structures subjected to impact." International Journal for Numerical and Analytical Methods in Geomechanics, 31: 14771515.

Peila, D., Oggeri, C., and Castiglia, C. (2007). "Ground reinforced embankments for rockfall protection: design and evaluation of full scale tests." Landslides, 4: 255-265.

RiskYdrogeo. (2006). "Risques hydro-géologiques en montagne : parades et surveillance", Projet 179 INTERREG IIIA, Technical report, http://www;risknat.org/projets/riskydrogeo (in french). 